\title{
POTENTIAL INFLUENCE FROM LOWER LIMB PREFERENCE AND 1-RM ON CARTILAGE THICKNESS
}

original paper

( ) University School of Physical Education in Wroclaw

DOI: https://doi.org/10.5114/hm.2021.100007

\section{RODRIGO BINI}

La Trobe Rural Health School, La Trobe University, Bendigo, Australia

\section{ABSTRACT}

Purpose. The aims of this study were to assess the potential retrospective relationship between limb preference and cartilage thickness, and to determine the association between measures of strength using one-repetition maximum (1-RM) tests and cartilage thickness.

Methods. A cross-sectional retrospective design was employed. Limb preference and injury history were collected with the Waterloo Inventory followed by ultrasound femoral cartilage imaging. Overall, 15 apparently healthy participants (11 males and 4 females) without musculoskeletal or neurological diseases volunteered for the study. After collection of anthropometric measures, warm-up, and familiarization, the participants performed 1-RM for back half-squat on a Smith machine. Ultrasound images were digitized to determine bilateral differences and femoral cartilage thickness.

Results. A significant moderate association between the existence of a prior injury and bilateral differences in cartilage thickness $(r=-0.63, p<0.01)$ in favour of the preferred limb was observed. There was a significant moderate association between 1-RM, as percentage of body mass, and the mean bilateral cartilage thickness $(r=0.58, p=0.049)$.

Conclusions. Bilateral differences in cartilage thickness are not associated with limb preference but depend on the history of lower limb injuries. The relative load lifted during a 1-RM half-squat is associated with thicker femoral cartilages, which suggests that relative strength is an important measure of cartilage health.

Key words: asymmetries, ultrasonography, resistance training, knee joint

\section{Introduction}

Osteoarthritis (OA) is a degenerative disorder arising from the reduction in thickness and volume of articular cartilage in synovial joints. A previous study found that the articular cartilage undergoes atrophy and becomes thinner under chronic reduced loading conditions [1]. Cartilage thickness has been measured with a number of different techniques, including magnetic resonance imaging (MRI), radiography, computed tomography scan, and ultrasonography [2, 3]. Even though MRI enables the assessment of the whole cartilage, this method is expensive and time consuming compared with ultrasound, which has been proven valid to assess cartilage thickness [4]. Ultrasound has been shown to allow measurements with an error of 0.08-0.11 mm [5].

Asymmetrical strength has been linked to a vari- ety of pathological conditions. It has been suggested that unequal loading distribution increases the risk of OA in weight-bearing joints [6]. Bilateral limb deficits cause differences in maximal force generating capacities when the muscles are contracted alone or in combination with contralateral muscles $[7,8]$. These imbalances are important to understand as muscle weakness can be a cause of musculoskeletal disorders [8]. Moreover, lower limb preference has been associated with asymmetries in lower limb force and power [9]. This relationship suggests that the limb subject to less load could be at risk of developing early OA because hyaline cartilages need moderate mechanical stress for maintenance of chondrocytes function [10,11].

Maintaining muscular strength is important to sustain health status and minimize the risk of injuries or disease [12]. From a cartilage health perspective, mechanical stress is critical if the load is within a physio-

Correspondence address: Rodrigo Bini, La Trobe Rural Health School, La Trobe University, Flora Hill Campus,

Postcode: 3550, Bendigo, Australia, e-mail: r.bini@latrobe.edu.au

Received: February 4, 2020

Accepted for publication: May 25, 2020

Citation: Bini R. Potential influence from lower limb preference and 1-RM on cartilage thickness. Hum Mov. 2021;22(2):9-15; doi: https://doi.org/10.5114/hm.2021.100007. 
logical load range [13]. Tuna et al. [14] observed that patients with knee OA showed increases in cartilage thickness when enrolled in a knee strengthening program. However, although muscle mass has been shown to predict the loss in cartilage volume [15], it would be beneficial to determine if objective measures of overall strength are associated with cartilage health. The gold standard to assess muscular strength is the isokinetic dynamometry. However, owing to the cost and operational requirements in using isokinetic dynamometry in the clinical space, one-repetition maximum (1-RM) testing is more often employed in the field [16]. As the 1-RM test allows the measurement of the maximum weight an individual can lift for one repetition maintaining correct form, it is often used to evaluate the effectiveness of training interventions [17]. With this in mind, it would be beneficial to determine if the 1-RM test is capable to reflect the link between muscle mass and cartilage thickness shown in prior research [15].

Therefore, the aims of this study were (1) to assess the potential relationship between leg preference, history of injury, and cartilage thickness; and (2) to determine the association between measures of strength using 1-RM tests and cartilage thickness.

\section{Material and methods}

\section{Study design}

This pilot study used a cross-sectional retrospective design to assess lower limb preference and the existence of prior lower limb injury that could affect cartilage thickness and how overall body strength (assessed from the 1-RM) would be associated with cartilage thickness. The participants attended one laboratory session which involved scanning their knees, collecting data on limb preference and the existence of prior injury, familiarizing with the exercise testing, and performing the 1-RM test.

\section{Participants}

Fifteen apparently healthy participants (11 males and 4 females) without existing musculoskeletal or neurological diseases volunteered for the study. At the time of the study, they were $23 \pm 5.5$ years old, with $74 \pm 10.7 \mathrm{~kg}$ of body mass and $174 \pm 9.1 \mathrm{~cm}$ of stature. After being provided information on the study and consenting to participate, 13 subjects (9 males and $4 \mathrm{fe}-$ males) engaged in the assessment of 1-RMs.

\section{Assessment of leg preference}

Limb preference and the existence of prior injury were assessed with the Waterloo Footedness Questionnaire [18]. This questionnaire was employed to allow us to convert measures of cartilage thickness into data for preferred and non-preferred limbs and to determine whether the participants had had a lower limb injury in the past. Ultrasound images were then assigned depending on limb preference. The questionnaire required each participant to imagine themselves performing a series of lower limb activities. There were 12 questions in total. The first question asked the participant to specify which lower limb they considered as their preferred, left or right. This was followed by 10 questions which assessed 2 types of tasks: mobilizing tasks, such as picking up a marble or kicking a ball, and stabilizing tasks, such as standing on one limb or balancing on a rail track. Question 11 ascertained whether the participant had had a previous injury that might have affected their limb preference, and question 12 queried whether they had previous training to encourage the use of a particular foot for an activity. The footedness questionnaire scored as follows: responses of (i) left always, (ii) left usually, (iii) equal, (iv) right usually, (v) right always were scored on a scale of $1-5$, with 1 standing for left always, 5 for right always, and 3 for equal.

\section{Ultrasound measurements}

An ultra-sonic diagnostic imaging system (DP-6900, Shenzhen Mindray Bio-Medical Electronics Co., Ltd., China) with a linear probe $(55 \mathrm{~mm}, 75 \mathrm{~L} 53 \mathrm{EA}-7.5 \mathrm{MHz}$, Shenzhen Mindray Bio-Medical Electronics Co., Ltd., China) was used to capture images from the anterior portion of the femoral cartilage (Figure 1).

Water-soluble transmission gel was applied to the scan site without depressing the skin. The probe scanner site was placed in the axial plane on the suprapatellar area in order to align the scan site with the anterior condyles of the femur, similar to prior studies $[5,19]$. For capturing images, the participants laid in dorsal decubitus on a massage table with both knees at maximum flexion. Two images were captured per knee at the participants' arrival in the laboratory (baseline).

\section{1-RM tests}

After ultrasound scanning, the participants performed 8-min walking on a treadmill at $5 \mathrm{~km} / \mathrm{h}$ of speed for general purpose warm-up. They then per- 


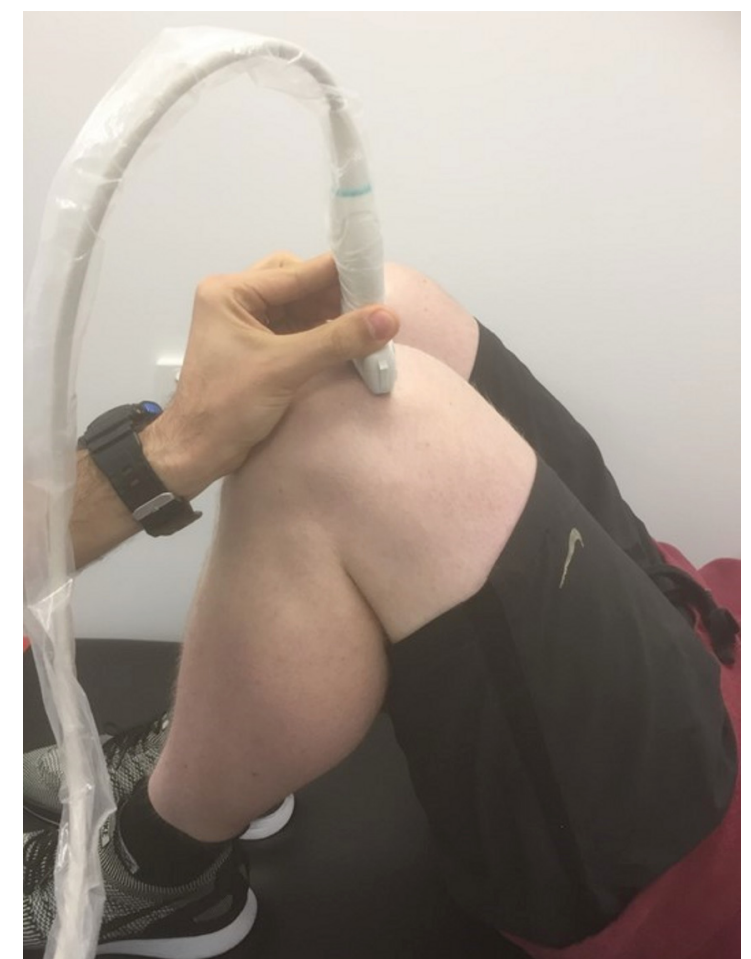

Figure 1. Ultrasound probe positioned aligned with the axial plane of the femur on the suprapatellar area with knees in maximum flexion

formed 1 set of 8 repetitions of body weight half-squats, for further warm-up, at the Smith machine exercise equipment (SCB1000, ProClub Line counter-balanced Smith machine, Body-Solid Inc., IL, USA). The Smith machine was then loaded with weights equivalent to approximately $50 \%$ of each participant's body mass and incremented in accordance with feedback from each participant on their perceived effort. A 6-20-point Borg scale [20] was used to rank the perceived effort from 'no exertion at all' to 'maximum exertion'. Once the participants achieved concentric failure with the attempted load within a maximum of 5 sets, the 1-RM load was determined. Two minutes of rest were provided between sets to minimize fatigue effects. Movement patterns were visually assessed by 2 experienced exercise scientists to ensure that each participant achieved approximately $90^{\circ}$ of knee flexion at the bottom of the half-squat. If the 1-RM was not reached in the 5 attempts, the participant had to attend an additional session at least 48 hours later to try again.

\section{Analysis of ultrasound images}

All ultrasound images were analysed by one trained rater using Image J (v.1.52a, NIH, USA). The analyses involved determining a straight line drawn from the cartilage-bone interface to the synovial space-cartilage,

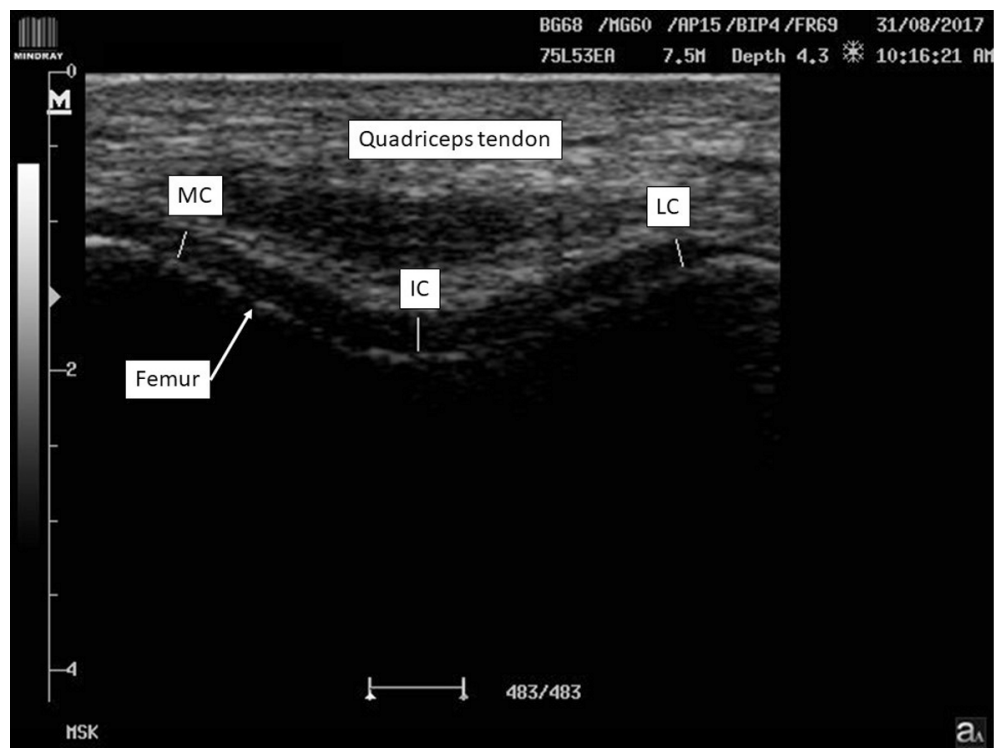

Figure 2. Ensemble illustration of the analysis of an ultrasound image from the knee. Quadriceps tendon and femur are shown along with the thickness of the cartilage for the medial condyle (MC), intercondylar grove (IC), and lateral condyle (LC)

representing the thickness of the anterior portion of the femoral cartilage. This distance was registered twice for the lateral femoral condyle, intercondylar grove, and medial femoral condyle [21], as illustrated in Figure 2.

\section{Statistical analysis}

A mean value for cartilage thickness was computed from the lateral femoral condyle, intercondylar grove, and medial femoral condyle and assessed for normality of distribution (using Shapiro-Wilk test) along with 1-RM, total load (1-RM + body mass), and 1-RM as a percentage of body mass. For assessing the relationship between lower limb preference and the existence of prior injury with bilateral differences in cartilage thickness, a Stepwise Multivariate Linear Regression was employed including the percentage difference between the preferred and non-preferred limbs for cartilage thickness (dependent variable) and independent variables such as the mean score from the 10 primary questions from the Waterloo Questionnaire, the scores provided for questions 11 (previous injury) and 12 (changes in leg preference through training), age, body mass and stature. Pearson correlations were calculated to further assess the relationship between each independent variable and dependent variable. Cohen's effect size $(d)$ [22] was utilized to compare bilateral differences in cartilage thickness for participants who indicated a prior injury vs. those without a history of injury and to compare participants who were encouraged to 


\section{HUMAN MOVEMENT}

R. Bini, Potential influence from lower limb preference and 1-RM on cartilage thickness

use a particular foot for an activity. The magnitude of differences was explored with Cohen's effect sizes $(d$, calculated as a ratio between the difference in mean values and the pooled standard deviation) and deemed meaningful when $d>0.80$ [22].

For assessing the relationship between measures of strength using 1-RM tests and cartilage thickness, a second Stepwise Multivariate Linear Regression was employed including the mean measure of cartilage thickness between limbs (dependent variable) and independent variables such as gender, age, stature, 1-RM, total load (1-RM + body mass), and 1-RM as a percentage of body mass. Pearson correlations were calculated to further assess the relationship between each independent variable and dependent variable. All analyses were conducted with IBM SPSS Statistics for Windows (version 25.0., IBM Corp., Armonk, NY, USA). Correlation coefficients were ranked in accordance with Dancey and Reidy [23] (i.e. $r=1.0$ indicates perfect association, $r$ between 0.9 and 0.69 indicates strong association, $r$ between 0.4 and 0.69 indicates moderate association, and $r$ smaller than 0.39 indicates small to none association).

\section{Ethical approval}

The research related to human use has complied with all the relevant national regulations and institutional policies, has followed the tenets of the Declaration of Helsinki, and has been approved by the university ethics committee (HEC17-028).

\section{Informed consent}

Informed consent has been obtained from all individuals included in this study.

\section{Results}

\section{Leg preference and injury}

Three participants indicated left leg preference, with 2 of them showing a mean score in the questionnaire larger than 3 points ('equal' in the questionnaire scale). The mean $(S D)$ score was 3.71 (0.73), with 3 participants obtaining less than 3 points (i.e. left leg preference). Three participants indicated that they had changed their leg preference because of injury and 3 others informed that they had changed their leg preference due to training requirements.

\section{Leg preference vs. cartilage thickness}

The mean $(S D)$ cartilage thickness for the preferred limb was 2.29 (0.4) $\mathrm{mm}$ and 2.31 (0.4) $\mathrm{mm}$ for the non- preferred limb, with a difference of $0 \%(9.8 \%)$. The linear regression model indicated a significant moderate association between the existence of a prior injury and bilateral differences in cartilage thickness (Table 1) in favour of the non-preferred limb. Bilateral differences in cartilage thickness for participants who had a prior injury were larger (11 $\pm 9 \%$, favouring the preferred limb) compared with those who never had an injury $(-1 \pm 10 \%$, favouring the non-preferred limb, $d=1.26$ ). Likewise, participants who were encouraged to use a particular foot for an activity had a larger bilateral difference in cartilage thickness $(8 \pm 7 \%$, favouring the preferred limb) compared with others $(-1 \pm 11 \%$, favouring the non-preferred limb, $d=1.01$ ). None of the other independent variables showed a significant association with bilateral differences in cartilage thickness (Table 1).

Table 1. Mean $(S D)$ results for independent variables included in the multivariate linear model with resulting $p$ value and Pearson correlation for their relationship with bilateral differences in cartilage thickness

\begin{tabular}{lccc}
\hline Independent variable & Mean $(S D)$ & $p$ & $\begin{array}{c}\text { Pearson } \\
\text { correlation }\end{array}$ \\
\hline Score for leg preference & $3.75(0.8)$ & 0.31 & -0.29 \\
Previous injury & $1.81(0.4)$ & $<0.01^{*}$ & -0.63 \\
Previous training & $1.81(0.4)$ & 0.06 & -0.50 \\
Age (years) & $24(6.9)$ & 0.27 & 0.45 \\
Body mass (kg) & $72(11.5)$ & 0.07 & 0.29 \\
Stature (cm) & $172(9.1)$ & 0.60 & 0.31 \\
\hline
\end{tabular}

* Significant association with bilateral differences in cartilage thickness.

Table 2. Mean $(S D)$ results for independent variables included in the multivariate linear model with resulting $p$ value and Pearson correlation for their relationship with mean bilateral cartilage thickness

\begin{tabular}{lccc}
\hline Independent variable & Mean $(S D)$ & $p$ & $\begin{array}{c}\text { Pearson } \\
\text { correlation }\end{array}$ \\
\hline Gender & $\begin{array}{c}\text { Not } \\
\text { applicable }\end{array}$ & 0.75 & -0.52 \\
\hline 1-RM (kg) & $102(34)$ & 0.84 & 0.50 \\
Total load (kg) & $176(41)$ & 0.81 & 0.42 \\
1-RM (\% body mass) & $137(39)$ & $0.049 *$ & 0.58 \\
Age (years) & $22(5.2)$ & 0.60 & -0.12 \\
Body mass (kg) & $74(11.1)$ & 0.78 & -0.01 \\
Stature (cm) & $172(8.5)$ & 0.39 & -0.09 \\
\hline
\end{tabular}

* Significant association with mean bilateral knee cartilage thickness. 
1-RM vs. cartilage thickness

The mean $(S D)$ cartilage thickness for both limbs was $2.28(0.3) \mathrm{mm}$. The linear regression model indicated a significant moderate association between the 1-RM as percentage of body mass and the mean bilateral cartilage thickness (Table 2, Figure 3). None of the other independent variables showed a significant association with cartilage thickness (Table 2).

\section{Discussion}

This study assessed the influence from limb preference on bilateral differences in cartilage thickness and determined the relationship between overall body strength and cartilage thickness. Findings from the data indicate that limb preference is not significantly associated with bilateral differences in femoral cartilage thickness and that body strength relative to body mass is moderately associated with femoral cartilage thickness. In addition, the existence of a prior injury seems to increase the bilateral asymmetry in cartilage thickness in favour of the preferred limb. These observations are new because no prior study has attempted to show the association between limb preference, overall body strength, and cartilage thickness as a strategy to prevent long-term reductions in cartilage thickness.

\section{Leg preference vs. cartilage thickness}

Bilateral asymmetry has been shown to affect tendon stiffness in favour of the dominant/preferred limb [24] but differences in cartilage thickness between limbs have not been associated with limb preference in a prior study [25]. The reason for tendon adaptations is due to the potentially increased load applied to the preferred limb during daily based activities [9]. For sustaining cartilage function, stress is important once applied within a physiological load range [13], which would suggest that a larger load applied to the preferred limb would lead to increased activity from the chondrocytes. This would then result in thicker cartilage in the preferred limb, which has not been shown true from current data and from a prior study [25]. The definition of lower limb dominance or footedness remains controversial. Previc's neurodevelopmental theory suggests that there is no dominant limb and that one foot is for mobilization and the other is for postural stability [26]. Although there was a favouring to the right limb in the current study among the participants, it should be acknowledged that the average limb preference result was also close to an equal limb preference score.

\section{1-RM vs Mean CT}

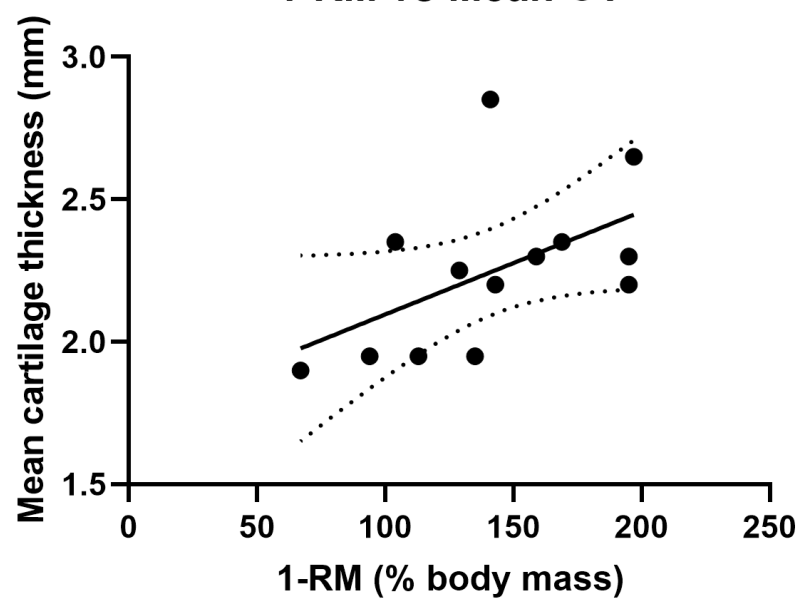

Figure 3. Scatter plot with linear regression and 95\% confidence interval for mean bilateral cartilage thickness (CT) as a function of one-repetition maximum (1-RM) loads

One possible explanation would be that the difference in load applied during daily activities could not be sufficient to trigger large changes in chondrocytes metabolism because of the low activity from these cells in comparison with tendons. McGrath et al. [27] completed a systematic review on functional lower limb dominance and found no significant result of limb dominance for any of the functional tests, which included isokinetic quadriceps and hamstring tests, single leg hop for distance, single leg vertical jump, and vertical ground reaction force. Therefore, it seems possible that the difference between limbs, in terms of force, may not have been enough to elicit large changes in cartilage thickness for a sample of apparently healthy individuals.

The existence of a prior injury, though, was moderately associated with bilateral differences in cartilage thickness. A meta-analysis conducted by Muthuri et al. [28] found that a previous knee injury was a major risk factor in developing knee OA. Therefore, it seems that the elements involved in the development of an injury (i.e. pain, loss of strength, etc.) are crucial for the function of the chondrocytes and, consequently, for cartilage thickness. Further studies are required to assess the time course of changes in cartilage thickness when knee pain or injury develops. This would help understand the role of various interventions for pain management in maintaining cartilage thickness.

\section{1-RM vs. cartilage thickness}

Overall body strength plays a key role in minimizing the risk for development of injuries and disease [12]. 
R. Bini, Potential influence from lower limb preference and 1-RM on cartilage thickness

However, although prior studies showed that participants with larger muscle mass presented thicker cartilage [15], data from the current study advance on that, indicating that overall strength measured with 1-RM from half-squats is also moderately associated with cartilage thickness. The half-squat is an important exercise that involves the recruitment of various muscles in the lower limbs and trunk. This exercise is part of regular prescription programs for improving strength and engages muscles associated with loading of the femoral cartilage (e.g. quadriceps and hamstrings). Interestingly, only the 1-RM load relative to body mass, not the total load, was associated with thicker cartilages. This result suggests that cartilages can adapt positively if load is applied on a regular basis (i.e. through resisted exercise) within a range that the person can manage. In other words, exercise load should be individualized to ensure that most benefits are obtained from training. Interestingly, prior evidence did not show benefits from endurance-related training (i.e. triathlon) in changing cartilage thickness [29]. Eckstein et al. [1], though, suggested that exercise training would not lead to thicker cartilage beyond a certain thickness as this would reduce the capacity for the cartilage to distribute stress.

As a preventive strategy, assessing overall body muscle mass [15] or thigh muscle mass [25] seems to be important to anticipate the risk of an early development of OA. Complementary measuring strength with exercise-specific tasks (i.e. half-squats) could be a viable strategy as this requires less expensive tools such as MRI scanners. In addition to regular strength assessments that form part of the exercise prescription program (e.g. maximum repetition tests), it could be implemented in order to anticipate the risk of early loss of cartilage function. Future studies should also consider evaluating unilateral strength (e.g. single leg halfsquats) in order to assess whether a given limb has an increased risk for developing OA owing to less strength. This is in line with the recommendations to assess contralateral limb mass and strength in patients with $\mathrm{OA}$ in order to anticipate the risk of a second disease [25].

\section{Limitations}

Data from this study were limited to a certain extent. As this was a pilot study, only 15 participants were included, with 13 performing the 1-RM tests. This limitation, though, provides the foundation for a follow-up study looking at a larger range of participants. Future studies could assess if improvements in cartilage thickness, as shown after a strength training program in patients with OA [14], could be observed in healthy participants as a preventive strategy to sustain cartilage health. This would further validate the association between relative strength and cartilage thickness as revealed in the present study. In addition, a wider range of strength tests (e.g. knee extensions, leg presses, etc.) could be conducted to assess which test is more strongly associated with cartilage thickness. A reduced number of participants reported the occurrence of an unspecified injury in their lower limbs in the past. This element should be further assessed in order to determine the influence of types and durations of injuries in cartilage thickness.

\section{Conclusions}

In summary, bilateral differences in femoral cartilage thickness were not associated with lower limb preference but depended on the existence of prior lower limb injuries. The relative load lifted during a 1-RM half-squat was associated with thicker femoral cartilages, which suggests that relative strength is an important measure of cartilage health.

\section{Acknowledgments}

The author thanks Ilena Karas, Dylan d'Souza, Kyle Morcombe, Gedd Hommelhoff, and Megan Lock for their assistance in data collection, as well as all participants who volunteered for this study.

\section{Disclosure statement}

The author does not have any financial interest and did not receive any financial benefit from this research.

\section{Conflict of interest}

The author states no conflict of interest.

\section{References}

1. Eckstein F, Hudelmaier M, Putz R. The effects of exercise on human articularcartilage. J Anat. 2006;208(4):491512; doi: 10.1111/j.1469-7580.2006.00546.x.

2. Keen HI, Wakefield RJ, Conaghan PG. A systematic review of ultrasonography in osteoarthritis. Ann Rheum Dis.2009;68(5):611-619; doi:10.1136/ard.2008.102434.

3. Chan WP, Lang P, Stevens MP, Sack K, Majumdar S, Stoller DW, et al. Osteoarthritis of the knee: comparison of radiography, CT, and MR imaging to assess extent and severity. Am J Roentgenol. 1991;157(4):799-806; doi: 10.2214/ajr.157.4.1892040.

4. Naredo E, Acebes C, Möller I, Canillas F, de Agustín JJ, de Miguel E, et al. Ultrasound validity in the measurement of knee cartilage thickness. Ann Rheum Dis. 2009;68(8):1322-1327; doi: 10.1136/ard.2008.090738. 
5. Harkey MS, Blackburn JT, Davis H, Sierra-Arévalo L, Nissman D, Pietrosimone B. Ultrasonographic assessment of medial femoral cartilage deformation acutely following walking and running. Osteoarthritis Cartilage. 2017;25(6):907-913; doi: 10.1016/j.joca.2016.12.026.

6. Mills K, Hettinga BA, Pohl MB, Ferber R. Betweenlimb kinematic asymmetry during gait in unilateral and bilateral mild to moderate knee osteoarthritis. Arch Phys Med Rehabil. 2013;94(11):2241-2247; doi: 10.1016/j.apmr.2013.05.010.

7. Maly T, Zahalka T, Mala L, Cech P. The bilateral strength and power asymmetries in untrained boys. Open Med. 2015;10(1):224-232; doi: 10.1515/med-2015-0034.

8. Mokha M, Sprague PA, Gatens DR. Predicting musculoskeletal injury in National Collegiate Athletic Association Division II athletes from asymmetries and individual-test versus composite functional movement screen scores. J Athl Train. 2016;51(4):276-282; doi: 10.4085/1062-6050-51.2.07.

9. Bishop C, Turner A, Read P. Effects of inter-limb asymmetries on physical and sports performance: a systematic review. J Sports Sci. 2018;36(10):1135-1144; doi: 10.1080/02640414.2017.1361894.

10. Nomura M, Sakitani N, Iwasawa H, Kohara Y, Takano S, Wakimoto Y, et al. Thinning of articular cartilage after joint unloading or immobilization. An experimental investigation of the pathogenesis in mice. Osteoarthritis Cartilage. 2017;25(5):727-736; doi: 10.1016/j.joca.2016. 11.013.

11. Maldonado DC, Pereira da Silva MC, El-Razi Neto S, Rodrigues de Souza M, Rodrigues de Souza R. The effects of joint immobilization on articular cartilage of the knee in previously exercised rats. J Anat. 2013;222(5): 518-525; doi: 10.1111/joa.12036.

12. Garber CE, Blissmer B, Deschenes MR, Franklin BA, Lamonte MJ, Lee I-M, et al. Quantity and quality of exercise for developing and maintaining cardiorespiratory, musculoskeletal, and neuromotor fitness in apparently healthy adults: guidance for prescribing exercise. Med Sci Sports Exerc. 2011;43(7):1334-1359; doi: 10.1249/MSS.0b013e318213fefb.

13. Sanchez-Adams J, Leddy HA, McNulty AL, O'Conor CJ, Guilak F. The mechanobiology of articular cartilage: bearing the burden of osteoarthritis. Curr Rheumatol Rep. 2014;16(10):451; doi: 10.1007/s11926-014-0451-6.

14. Tuna S, Balcı N, Özçakar L. The relationship between femoral cartilage thickness and muscle strength in knee osteoarthritis. ClinRheumatol.2016;35(8):20732077; doi: 10.1007/s10067-016-3271-4.

15. Cicuttini FM, Teichtahl AJ, Wluka AE, Davis S, Strauss BJG, Ebeling PR. The relationship between body composition and knee cartilage volume in healthy, middle-aged subjects. Arthritis Rheum. 2005;52(2):461-467; doi: 10.1002/art.20791.

16. Verdijk LB, van Loon L, Meijer K, Savelberg HHCM. One-repetition maximum strength test represents a valid means to assess leg strength in vivo in humans. J Sports
Sci. 2009;27(1):59-68; doi: 10.1080/0264041080242 8089.

17. Braith RW, Graves JE, Leggett SH, Pollock ML. Effect of training on the relationship between maximal and submaximal strength. Med Sci Sports Exerc. 1993;25(1): 132-138; doi: 10.1249/00005768-199301000-00018.

18. Elias LJ, Bryden MP, Bulman-Fleming MB. Footedness is a better predictor than is handedness of emotional lateralization. Neuropsychologia. 1998;36(1):37-43; doi: 10.1016/s0028-3932(97)00107-3.

19. Kilic G, Kilic E, Akgul O, Ozgocmen S. Ultrasonographic assessment of diurnal variation in the femoral condylar cartilage thickness in healthy young adults. Am J Phys Med Rehabil. 2015;94(4):297-303; doi: 10.1097/ PHM.0000000000000179.

20. Borg GAV. Psychophysical bases of perceived exertion. Med Sci Sports Exerc. 1982;14(5):377-381; doi: 10.1249/ 00005768-198205000-00012.

21. Malas FÜ, Kara M, Aktekin L, Ersöz M, Özçakar L. Does vitamin D affect femoral cartilage thickness? An ultrasonographic study. Clin Rheumatol. 2014;33(9): 1331-1334; doi: 10.1007/s10067-013-2432-y.

22. Cohen J. Statistical power analysis for the behavioral sciences. New York: Lawrence Erlbaum Associates; 1988.

23. Dancey CP, Reidy J. Statistics without maths for psychology with psychology dictionary. Harlow: Prentice Hall; 2004.

24. Bohm S, Mersmann F, Marzilger R, Schroll A, Arampatzis A. Asymmetry of Achilles tendon mechanical and morphological properties between both legs. Scand J Med Sci Sports. 2015;25(1):e124-e132; doi: 10.1111/ sms.12242.

25. Eckstein F, Müller S, Faber SC, Englmeier K-H, Reiser M, Putz R. Side differences of knee joint cartilage volume, thickness, and surface area, and correlation with lower limb dominance - an MRI-based study. Osteoarthritis Cartilage. 2002;10(12):914-921; doi: 10.1053/joca.2002. 0843.

26. Sadeghi H, Allard P, Prince F, Labelle H. Symmetry and limb dominance in able-bodied gait: a review. Gait Posture. 2000;12(1):34-45; doi: 10.1016/s0966-6362 (00)00070-9.

27. McGrath TM, Waddington G, Scarvell JM, Ball NB, Creer R, Woods K, et al. The effect of limb dominance on lower limb functional performance - a systematic review. J Sports Sci. 2016;34(4):289-302; doi: 10.1080/ 02640414.2015 .1050601$.

28. Muthuri SG, McWilliams DF, Doherty M, Zhang W. History of knee injuries and knee osteoarthritis: a metaanalysis of observational studies. Osteoarthritis Cartilage. 2011;19(11):1286-1293; doi: 10.1016/j.joca. 2011. 07.015 .

29. Eckstein F, Faber S, Mühlbauer R, Hohe J, Englmeier K-H, Reiser M, et al. Functional adaptation of human joints to mechanical stimuli. Osteoarthritis Cartilage. 2002;10(1):44-50; doi: 10.1053/joca.2001.0480. 